\section{In vivo detection of clinically non-apparent ocular surface inflammation in patients with meibomian gland dysfunction-associated refractory dry eye symptoms: a pilot study}

\author{
Abstract \\ Purpose The utility of in vivo confocal \\ microscopy (IVCM) in the investigation of \\ palpebral conjunctival and corneal \\ inflammation in patients with meibomian \\ gland dysfunction (MGD)-associated refractory \\ dry eye symptoms following gland expression, \\ despite objective clinical improvement. \\ Methods A retrospective, observational pilot \\ study was conducted evaluating five patients \\ with MGD-associated refractory dry eye \\ symptoms and three control groups: \\ symptomatic untreated MGD patients $(n=3)$, \\ treatment-responsive MGD patients with \\ improved symptoms $(n=3)$ and asymptomatic \\ healthy normals $(n=11)$. Ocular surface disease \\ index (OSDI) scores, tear break-up time \\ (TBUT), the number of meibomian glands \\ yielding liquid secretion (MGYLS), palpebral \\ conjunctival epithelial and substantia propria \\ immune cell (EIC, SIC), and corneal dendritic \\ cell (DC) densities were measured. \\ Results Despite clinical improvement \\ (TBUT: $6.4 \pm 1.2$ s to $10.1 \pm 2.1 \mathrm{~s}, P=0.03$; \\ MGYLS: $3.5 \pm 0.8$ glands to $7.0 \pm 1.1$ glands, \\ $P=0.13)$ and a normal clinical examination \\ post treatment, MGD patients remained \\ symptomatic. IVCM revealed increased \\ immune cells in the palpebral conjunctiva \\ (refractory MGD EIC $=592.6 \pm 110.1$ cells/ \\ $\mathrm{mm}^{2}$; untreated MGD EIC $=522.6 \pm 104.7$ \\ cells $/ \mathrm{mm}^{2}, P=0.69$; responsive MGD \\ $\mathrm{EIC}=194.9 \pm 119.4 \mathrm{cells} / \mathrm{mm}^{2}, P<0.01 ;$ \\ normals $\mathrm{EIC}=123.7 \pm 19.2 \mathrm{cells} / \mathrm{mm}^{2}$,
}

Y Qazi ${ }^{1}$, A Kheirkhah¹, C Blackie², A Cruzat ${ }^{1}$, M Trinidad ${ }^{1}$, C Williams ${ }^{1}$, DR Korb ${ }^{2}$ and P Hamrah ${ }^{1}$
${ }^{1}$ Ocular Surface Imaging Center, Cornea Service, Massachusetts Eye and Ear Infirmary, Department of Ophthalmology, Harvard Medical School, Boston, MA, USA

${ }^{2}$ Korb Associates, Boston, MA, USA

MGD DC $=60.9 \pm 28.3 \mathrm{cells} / \mathrm{mm}^{2}$; normals $\mathrm{DC}=25.9 \pm 6.3$ cells $\left./ \mathrm{mm}^{2} ; P=0.43\right)$. EIC did not correlate with TBUT $\left(R_{\mathrm{s}}=-0.26, P=0.33\right)$. OSDI scores correlated with both EIC $\left(R_{\mathrm{s}}=0.76, P<0.001\right)$ and TBUT $\left(R_{\mathrm{s}}=-0.69\right.$, $P<0.01)$ but not SIC. Intraglandular immune cells were also seen.

Conclusion MGD-associated refractory symptoms and the symptom-sign disparity may be explained by clinically non-apparent, active inflammation of the palpebral conjunctiva as detected by IVCM. These patients may benefit from anti-inflammatory therapy.

Eye (2015) 29, 1099-1110; doi:10.1038/eye.2015.103; published online 19 June 2015

\section{Introduction}

Meibomian gland dysfunction (MGD) is known to be the leading cause of evaporative dry eye disease (DED). ${ }^{1-3}$ Population-based studies have revealed that the prevalence of MGD is reported to be nearly $20 \%$ among Caucasians and $60 \%$ on average in Asian populations. ${ }^{4,5}$ Treatments for MGD typically combine a number of approaches ranging from lubrication, oral omega-3 fatty acids, and targeting clinically apparent inflammation with steroids and antibacterial therapy. ${ }^{6}$ Further, intraglandular obstruction, central to the pathogenesis of $\mathrm{MGD}^{7}$ may be reduced through self-administered heat compresses and lid massage, ${ }^{6}$ meibomian gland
Correspondence: P Hamrah, Ocular Surface Imaging Center, Cornea Service, Massachusetts Eye and Ear Infirmary,

Department of Ophthalmology, Harvard Medical School, 243 Charles Street, Boston, 02114 MA, USA Tel: +617 888 3363; Fax: +617 5734300 E-mail: pedram_hamrah@ meei.harvard.edu or p_hamrah@yahoo.com

Received: 23 September 2014

Accepted in revised form: 13 May 2015 Published online: 19 June 2015

This work was presented in part at the Association for Research in Vision and Ophthalmology in Ft. Lauderdale, Florida, May 2012; at the American Academy of Optometry in Boston, Massachusetts, October 2011; and at the 27th Biennial Cornea Conference in Boston, Massachusetts, September 2011 
expression in the clinic, or through newer advanced techniques such as thermal pulsation procedures, intraductal meibomian gland probing, and intense pulse light therapy performed in the outpatient setting. ${ }^{8-10}$ Although these newer technologies have met with improved outcomes in a subset of patients, ${ }^{8-10}$ there remains a cohort of patients whose treatment is complicated by persistent symptoms despite objective improvement in clinical signs. Symptom-sign disparity is a well-recognized challenge in the management of DED, as the symptoms remain unexplained by the lack of proportional findings on clinical examination and vice versa. ${ }^{11-15}$

To date, the management and treatment of inflammation in MGD have been addressed in the context of clinically apparent inflammation. Here, we employed high-magnification, laser-scanning in vivo confocal microscopy (IVCM) to examine the palpebral conjunctivae and corneas in a series of five such patients with MGD that showed clear improvement on clinical examination following mechanical gland expression, yet had refractory symptoms of ocular discomfort. Although IVCM has already been established as a sensitive and specific tool in the diagnosis of MGD, including its ability to detect palpebral conjunctival inflammatory cells, ${ }^{16}$ herein, we demonstrate that in comparison to controls, MGD patients with refractory symptoms had significant increase in inflammatory cells of the palpebral conjunctiva but not the cornea. Unlike previous work done in the field, our pilot study identifies clinically non-apparent inflammation as a probable explanation for refractory symptoms in MGD, providing a rationale for symptom-sign disparity. Furthermore, this pilot study also highlights the possible benefit from anti-inflammatory therapy in MGD patients with refractory symptoms following mechanical and thermal gland expression.

\section{Materials and methods}

\section{Study design and patient population}

We conducted a pilot, institutional review boardapproved, retrospective, observational study, investigating both eyes of five patients with MGDassociated persistent symptoms $(41.8 \pm 6.6$ years; 4 females:1 male). Three age- $(P=0.11)$ and sex-matched $(P=0.42)$ control groups were used in this study, analyzing both eyes of the MGD subjects and one eye of healthy individuals: (a) positive control group comprising three untreated, symptomatic MGD subjects with both symptoms and clinical signs (54.0 \pm 3.3 years; 3 females), (b) a treated control group of three MGD subjects (54.0 \pm 3.3 years; 3 females) with improved symptoms and signs who had been treated with a systemic antiinflammatory drug (doxycycline $200 \mathrm{mg}$ BID) with or without a topical anti-inflammatory agent (azithromycin, corticosteroids), and (c) a negative control group of 11 normal, healthy asymptomatic individuals (37.2 \pm 3.3 years; 6 females:5 males). The patients with refractory symptoms had a clinical diagnosis of MGD, and were referred to the Ocular Surface Imaging Center at the Massachusetts Eye and Ear Infirmary (MEEI), Boston, Massachusetts, in 2011 for the evaluation of persistent symptoms despite objective clinical improvement in both tear break-up time (TBUT) and the number of meibomian glands yielding liquid secretion (MGYLS) score post treatment (Table 1). The patients had been diagnosed with MGD based on clinical symptoms of DED, clinical slitlamp examination with a TBUT of less than $10 \mathrm{~s}$, with or without a reduction in the MGYLS score of less than 6 patent glands. These patients had persistence of dry eye symptoms, despite receiving treatments including meibomian gland expression with or without thermal eyelid pulsation (LipiFlow, TearScience, Morrisville, NC, USA), in combination with conservative management of warm compresses, lid scrubs, artificial tears, and a lubricating ointment at night.

Medical charts and study forms were reviewed for medical history, and details of anterior segment slit-lamp examination findings measuring corneal fluorescein staining, TBUT, and the MGYLS score. Patient symptom severity scores from the OSDI $^{17-19}$ and Standard Patient Evaluation of Eye Dryness (SPEED) questionnaires were also reviewed. ${ }^{20}$ IVCM images of the palpebral conjunctiva and central cornea were then analyzed by two masked observers.

\section{In vivo confocal microscopy}

IVCM (Heidelberg Retinal Tomograph 3 with the Rostock Cornea Module, Heidelberg Engineering $\mathrm{GmbH}$, Heidelberg, Germany) images of the palpebral conjunctiva and cornea for all subjects were reviewed for analysis. IVCM had been performed on both eyes of patients with MGD-associated persistent dry eye symptoms, and on one randomly selected eye of controls, as previously described. ${ }^{21}$ IVCM of the palpebral conjunctiva had been performed on the center of both upper and lower everted eyelids, approximately half way between the eyelid fold and the eyelid margin. A total of four to eight sequence scans obtained were reviewed per eyelid. The images had sampled the eyelid adequately by scanning across the eyelid from the center, across nasally and temporally, acquiring images from the epithelium, through the substantia propria (stroma), typically at a range of depths from 5-200 $\mu \mathrm{m}$, or until the tarsal plate was approached, indicated by visualization of a dark, amorphous background. After imaging the palpebral conjunctiva, IVCM images of the central cornea had been acquired as a series of two to four sequence scans, with 
particular focus on epithelial dendritic cells (DCs), found typically at a depth of $50-80 \mu \mathrm{m}$.

\section{Image analysis}

All measurements were made using Image software (National Institutes of Health, Bethesda, MD, USA; available at http:/ /rsb.info.nih.gov.ezp-prod1.hul.harvard. edu/ij/http:/ /rsb.info.nih.gov.ezp-prod1.hul.harvard. $\mathrm{edu} / \mathrm{ij} /$ ). Representative images were selected by a single observer for analysis by two masked observers. The criteria for selection of images included good focus for structures of interest, visualization of the structure(s) of interest, absence of motion artifacts, and without regions of overexposure or hyperreflectance that may make differentiating structures difficult. Three images per parameter per eye were analyzed for each subject by two masked observers. For patients, measurements from both eyes of each patient were averaged to represent a single sample. All results were reported as a mean of the measurements made by both the observers and expressed as cell density (cells $\left./ \mathrm{mm}^{2}\right) \pm$ standard error of mean (SEM).

Conjunctiva Palpebral conjunctival images were analyzed for epithelial and stromal (substantia propria) immune cell densities. Other groups have quantified epithelial immune cells at the eyelid margin, ${ }^{16,22}$ and the mucosa using pre-installed software (Cell Count, Heidelberg Engineering GmbH, Germany). ${ }^{23,24}$ We imaged the palpebral conjunctival epithelium and substantia propria (stroma), performing quantitation using ImageJ (National Institutes of Health) and incorporated measures to prevent overestimation of cell density. Therefore, our method of quantifying immune cell density, while comparable to some studies, ${ }^{24}$ has been defined as follows. Briefly, for en face images, the whole frame $\left(400 \times 400 \mu \mathrm{m}^{2}\right)$ was analyzed, counting all cells within the frame with the exclusion of cells along the superior and right-hand borders of the frame to prevent overestimation in our analysis. Density was expressed as the number of cells per millimeter square. For oblique images, immune cells were counted only within the region of interest. The region of interest was selected and measured using the polygon tool in Image J. Immune cells were identified as hyperreflective (nearly white), polymorphous (dendritiform, non-dendritiform, spindleshaped) cellular structures within the epithelium and stroma, ranging in size from 5 to $20 \mu \mathrm{m}$.

Epithelial immune cell (EIC) density: In the analysis of EIC, particular attention was paid to avoid counting goblet cells and epithelial cells that are morphologically different and less hyperreflective than immune cells as described in previous IVCM studies. ${ }^{24,25}$ As compared with immune cells, goblet 
cells were identified as larger $(\sim 30 \mu \mathrm{m})$, uniformly ovoid and less hyperreflective cells in the epithelium. ${ }^{23,24}$

Stromal immune cell (SIC) density: In the analysis of SIC, particular attention was paid not to count immune cells within glandular structures, blood and lymphatic vessels, as they were not within the stromal matrix of the palpebral conjunctiva.

Cornea IVCM images at the level of basal epithelial layers, basal lamina, or subbasal nerve plexus were chosen for the quantification of DC density. Analysis was performed as previously described. ${ }^{26}$ Briefly, the whole frame was analyzed and DCs were morphologically identified as hyperreflective dendritiform structures with cell bodies that allowed us to differentiate these structures from the corneal nerves.

\section{Statistical analysis}

Normality of data was determined using the ShapiroWilk normality test based on which either parametric (Student's $t$-test, one-way analysis of variance) or nonparametric tests (Mann-Whitney $U$-test, Kruskal-Wallis with post hoc tests) were applied for inter-group comparisons. Based on normality of data, Spearman's rank-order correlation coefficient $\left(R_{\mathrm{S}}\right)$ was used to determine correlation between imaging parameters (EIC, DC), symptoms (OSDI scores), and clinical metrics of assessment (TBUT). Data from the worse eye were used for correlations with OSDI scores. Inter-observer agreement and reliability were determined using the intraclass correlation coefficient (ICC) for each measured parameter (EIC, SIC, DC). Furthermore, inter-observer variability was also determined for both the conjunctival (EIC, SIC) and corneal (DC) imaging parameters by calculating the mean difference in absolute measurements (bias) and mean percentage difference in measurements between the two observers.

\section{Statement of ethics}

We certify that all applicable institutional and governmental regulations concerning the ethical use of human volunteers were followed during this research.

\section{Results}

\section{Clinical symptom-sign disparity}

Following mechanical treatment in the refractory MGD group, while clinical signs improved to within normal limits (pre-treatment TBUT $=6.4 \pm 1.2 \mathrm{~s}$, post-treatment
TBUT $=10.1 \pm 2.1 \mathrm{~s}, P=0.03$; pre-treatment MGYLS score $=3.5 \pm 0.8$ glands, post-treatment MGYLS score $=7.0 \pm 1.1$ glands, $P=0.14$, Table 1$)$, moderate to severe symptoms continued to persist (pre-treatment OSDI score $=41.7 \pm 9.4$, post-treatment OSDI score $=32.9 \pm 9.1$, $P=0.18$; pre-treatment SPEED score $=15.8 \pm 2.3$, posttreatment SPEED score $=10.4 \pm 2.7, P=0.07$, Table 1 ), underscoring the disparity between post-treatment clinical signs and symptom severity.

\section{IVCM of the palpebral conjunctiva}

Comparison of refractory MGD patients to normals Gross structural changes of meibomian glands can be appreciated at the eyelid margin on slit-lamp bio-microscopy. Figure 1 shows representative slit-lamp photographs of a healthy eyelid margin (Figure 1a) and in MGD (Figure 1b), showing an irregular conjunctival fold due to pouting of meibomian glands. As compared with healthy subjects (Figure 1c and f), patients with refractory symptoms had increased densities of palpebral conjunctival EIC and SIC distinctly evident on IVCM micrographs (Figure 1d and g), suggesting some infiltration of inflammatory cells in the deeper layers of the palpebral conjunctiva as well. Patients with refractory symptoms had epithelial immune cell densities nearly five-fold greater than healthy eyes of controls (refractory MGD EIC $=592.6 \pm 110.1$ cells $/ \mathrm{mm}^{2}$, normals EIC $=123.7 \pm 19.2$ cells $/ \mathrm{mm}^{2}, P<0.001, n=16$, Figure $1 \mathrm{e}$ ) with a near three-fold increase in stromal immune cell density as well (refractory MGD SIC $=93.9 \pm 44.2$ cells $/ \mathrm{mm}^{2}$, normals SIC $=38.8 \pm 9.5$ cells $/ \mathrm{mm}^{2}, P=0.11$, $n=16$, Figure $1 \mathrm{~h})$. Corroborating and consistent with the symptom-sign disparity in refractory MGD, EIC did not correlate significantly with TBUT $\left(R_{\mathrm{S}}=-0.26, P=0.33\right.$, $n=16$, Figure 1i). OSDI scores showed a highly significant positive correlation with EIC $\left(R_{\mathrm{S}}=0.76, P<0.001, n=16\right.$; Figure $1 j)$ but not SIC $\left(R_{\mathrm{S}}=-0.17, P=0.53, n=16\right)$ and an inverse correlation with TBUT $\left(R_{\mathrm{s}}=-0.69, P=0.003\right.$, $n=16$ ). Additional indicators of disease in the substantia propria included the presence of dilated glands in refractory patients (Figure 11), suggestive of obstruction along the ductal system, unlike healthy eyes where ductal dilatation was not seen (Figure 1k).

\section{Comparison of refractory MGD, untreated symptomatic MGD, and treatment-responsive MGD patients}

In vivo confocal micrographs of MGD patients with refractory symptoms showed dense infiltration of immune cells in the palpebral conjunctival epithelium (Figure 2a) similar to untreated MGD patients (Figure 2b), and in stark contrast to the lower density of immune cells seen in treatmentresponsive MGD patients with improved symptoms 

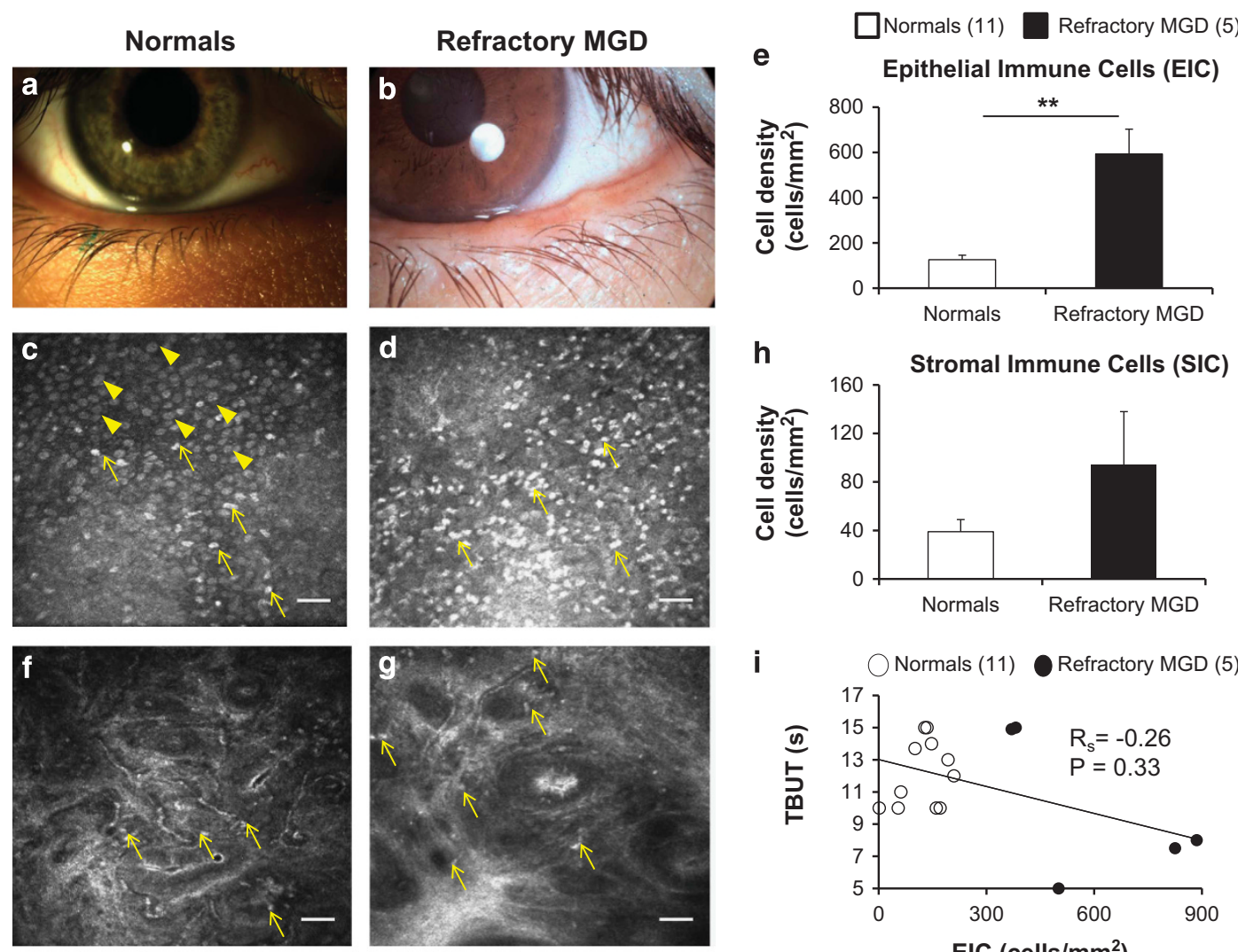

i
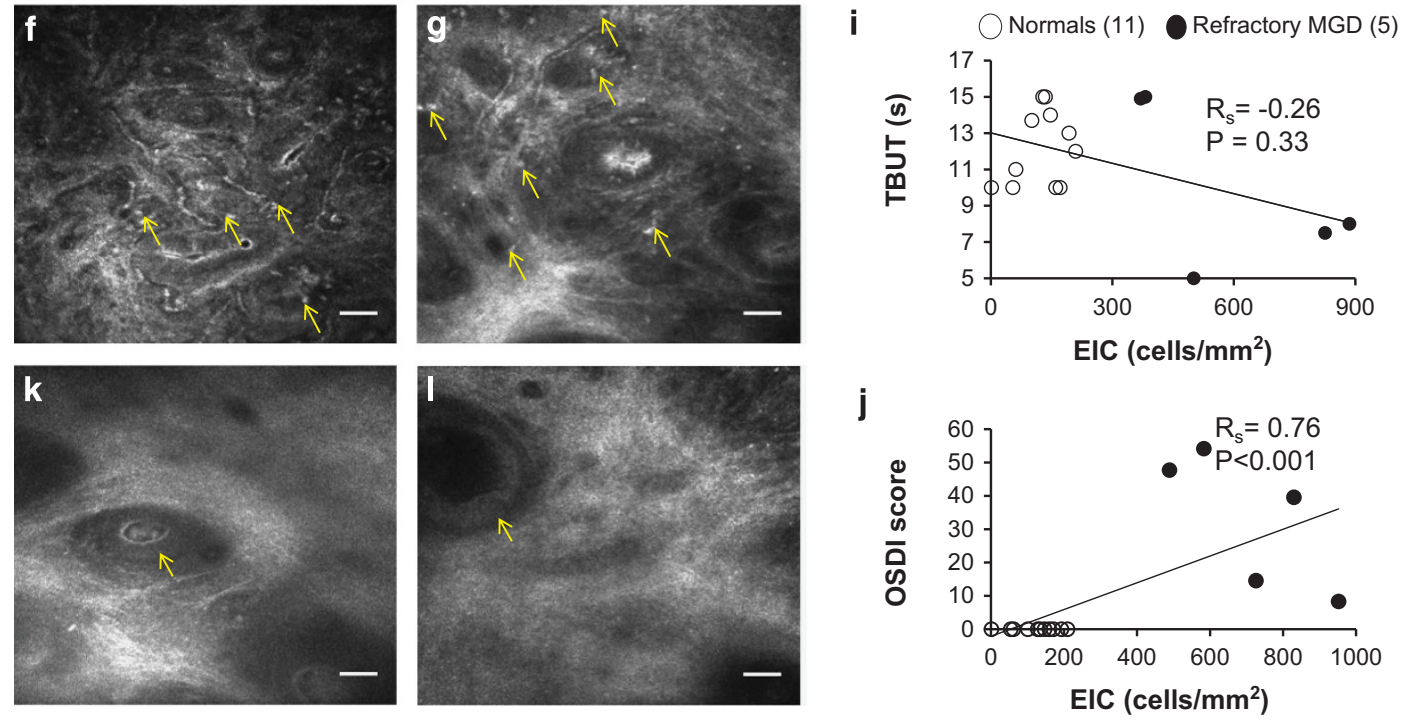

Figure 1 Comparison of refractory MGD patients with healthy normal controls on in vivo confocal microscopy. Representative slitlamp photographs of the eyelid margin in healthy eyes (a) and patients with MGD (b), showing plugging and pouting of meibomian glands in MGD (b). As compared with eyes of healthy, normal, asymptomatic controls (c, f; arrows) with numerous goblet cells (c, arrowheads), en face corneal in vivo confocal micrographs (HRT 3/RCM, Heidelberg Engineering, Germany) of MGD patients with refractory symptoms demonstrated increased infiltration of immune cells ( $\mathrm{d}, \mathrm{g}$; arrows) in both the conjunctival epithelium $\left(\mathrm{EIC}=592.6 \pm 110.1\right.$ cells $/ \mathrm{mm}^{2}$ vs $123.7 \pm 19.2$ cells $\left./ \mathrm{mm}^{2} P<0.01 ; \mathrm{d}, \mathrm{e}\right)$ and stroma $\left(\mathrm{SIC}=93.9 \pm 44.2 \mathrm{cells} / \mathrm{mm}^{2}\right.$ vs $38.8 \pm 9.5 \mathrm{cells} / \mathrm{mm}$, $P=0.11 ; \mathrm{g}, \mathrm{h})$. The near five-fold increase in conjunctival epithelial immune cell density (EIC; e) showed a trend of inverse correlation with tear break-up time (TBUT; $R_{\mathrm{S}}=-0.26, P=0.33, n=16$; i), and a highly significant positive correlation with symptom severity (OSDI scores; $\left.R_{\mathrm{S}}=0.76, P<0.001, n=16 ; \mathrm{j}\right)$ supporting the relationship between symptom severity in MGD and density of palpebral conjunctival EICs. In some healthy eyes, occasional intraglandular immune cells within ductules of presumed meibomian glands were observed ( $k$, arrow) without ductular dilatation (lumen diameter $<50 \mu \mathrm{m}$ ). Dilated glands can be seen in patients with refractory MGDassociated dry eye symptoms (l, arrow), suggestive of occult deep-seated glandular obstruction. Results are reported as mean \pm SEM. A probability value $(P)$ of less than 0.05 was considered statistically significant $\left(^{*}\right)$, whereas $P<0.01$ was considered highly statistically significant ${ }^{* *}$. Legend: numbers in parentheses represent the number of patients per group. Scale bar represents $40 \mu \mathrm{m}$.

(Figure 2c). However, stromal immune cells remained more numerable in refractory MGD patients (Figure 2d) in comparison to both untreated and treatment-responsive MGD patients (Figure 2e and $\mathrm{f}$ ) suggestive of deeper tissue inflammation in MGD patients with persistent dry eye symptoms. Anti-inflammatory treatment appeared to reduce the load of immune cells in both the conjunctival epithelium and stroma among treatment-responsive patients with improved symptoms (Figure 2c and f). Upon quantitation, patients with refractory symptoms had epithelial immune cell densities comparable to that of untreated symptomatic MGD patients (refractory MGD EIC $=592.6 \pm 110.1$ cells $/ \mathrm{mm}^{2}$, 
Refractory MGD
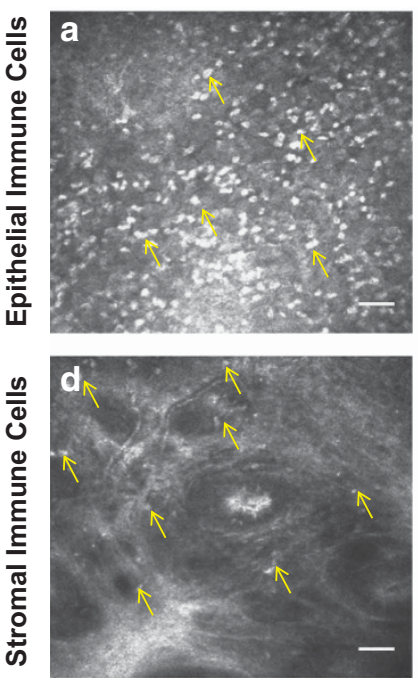

Untreated MGD
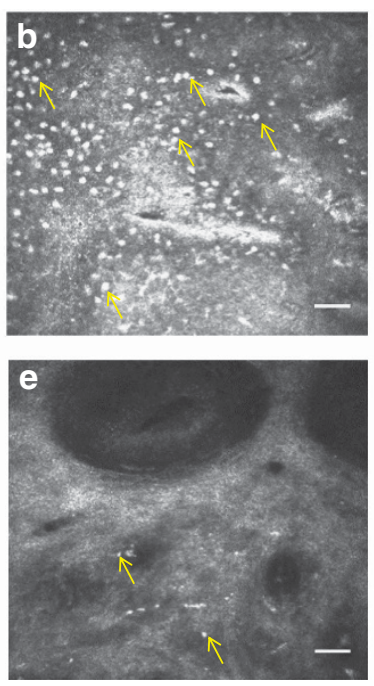

Responsive MGD
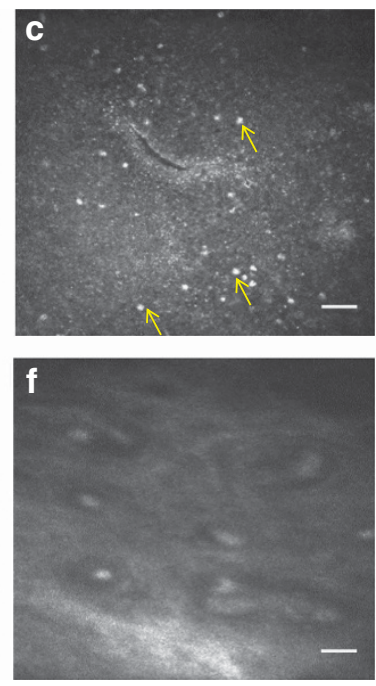

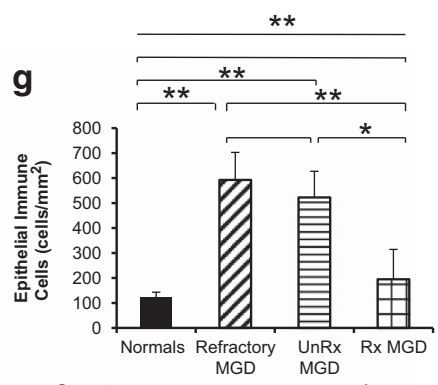

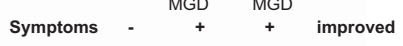

Signs $\quad-\quad+\quad+$ improved

h

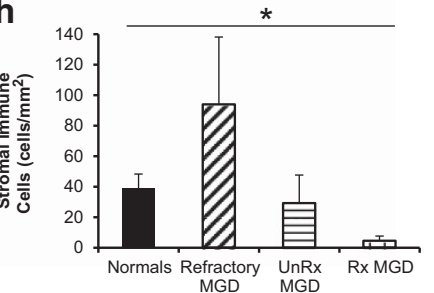

Figure 2 Comparison of refractory MGD, untreated symptomatic MGD, and treatment-responsive MGD patients on in vivo confocal microscopy. En face in vivo confocal micrographs (HRT 3/RCM, Heidelberg Engineering, Germany) of the palpebral conjunctiva showed increased infiltration of immune cells in both the conjunctival epithelium $(\mathrm{a}-\mathrm{c}, \mathrm{g}$; analysis of variance (ANOVA), $P<0.0001)$ and stroma ( $\mathrm{d}-\mathrm{f}$, h; ANOVA, $P=0.03$ ) of eyes with MGD-associated refractory dry eye symptoms. Epithelial immune cell density (EIC) in refractory patients $\left(592.6 \pm 110.1\right.$ cells $\left./ \mathrm{mm}^{2} ; \mathrm{a}, \mathrm{g}\right)$ was nearly five-fold greater than normals (EIC $=123.7 \pm 19.2 \mathrm{cells} / \mathrm{mm}^{2} P<0.01 ; \mathrm{g}$ ), comparable to epithelial inflammation in untreated, highly symptomatic MGD patients $\left(522.6 \pm 104.7\right.$ cells $\left./ \mathrm{mm}^{2}, P=0.69 ; \mathrm{b}, \mathrm{g}\right)$, and three-fold higher than that of treatment-responsive and less symptomatic MGD patients $\left(194.9 \pm 119.4\right.$ cells $/ \mathrm{mm}^{2}, P<0.01 ; \mathrm{c}$, g). Stromal immune cell density (SIC) was increased several-fold in refractory patients $\left(93.9 \pm 44.2\right.$ cells $\left./ \mathrm{mm}^{2} ; \mathrm{d}, \mathrm{h}\right)$ as compared with untreated, symptomatic MGD patients $\left(29.2 \pm 18.4\right.$ cells $\left./ \mathrm{mm}^{2}, P=0.33 ; \mathrm{e}, \mathrm{h}\right)$, and treatment-responsive, less symptomatic MGD patients $\left(4.6 \pm 3.1 \mathrm{cells} / \mathrm{mm}^{2}, P=0.18\right.$; $\mathrm{f}, \mathrm{h})$. Results are reported as mean \pm SEM. A probability value $(P)$ of less than 0.05 was considered statistically significant $\left(^{*}\right)$, whereas $P$ of less than 0.01 was considered highly statistically significant $\left(^{* *}\right)$. Numbers in parentheses represent the number of patients per group: normals (11), refractory MGD (5), unRx MGD (3), Rx MGD (3). Axes: untreated (UnRx), treatment-responsive (Rx).

untreated MGD EIC $=522.6 \pm 104.7$ cells $/ \mathrm{mm}^{2}, P=0.67$, Figure $2 \mathrm{~g}$ ) and nearly five-fold greater than healthy eyes of successfully treated MGD patients $(\mathrm{EIC}=194.9 \pm 119.4$ cells $/ \mathrm{mm}^{2}, P<0.01$, Figure $2 \mathrm{~g}$ ) indicating the presence of active superficial conjunctival inflammation. Upon advancing deeper into the tissue, a trend of increased conjunctival stromal immune cell density was observed in MGD patients with refractory symptoms as compared with both symptomatic, untreated and treatmentresponsive MGD patients (refractory MGD $\mathrm{SIC}=93.9 \pm 44.2 \mathrm{cells} / \mathrm{mm}^{2}$, untreated MGD $=29.2 \pm 18.4$ cells $/ \mathrm{mm}^{2}, P=0.33$, treatment-responsive MGD SIC $=4.6 \pm 3.1$ cells $/ \mathrm{mm}^{2}, P=0.18$, Figure $2 \mathrm{~h}$ ).

\section{IVCM of the cornea}

Slit-lamp biomicroscopy did not reveal corneal fluorescein staining in patients with MGD-associated refractory dry eye symptoms at the time of IVCM imaging. Figure 3a demonstrates the absence of corneal fluorescein staining in healthy eyes along with a stable tear film, which was comparable to eyes with refractory symptoms (Figure 3b). Consistent with clinical examination, IVCM did not show a significant difference in central corneal DC density between controls (Figure 3c and e; DCs $=25.9 \pm 6.3$ cells/ $\mathrm{mm}^{2}$ ) and patients (Figure $3 \mathrm{~d}$ and e; DCs $=60.9 \pm 28.3$ cells $/ \mathrm{mm}^{2}, P=0.43$ ). Furthermore, corneal DC density did not correlate with TBUT (Figure 3f).

\section{Inter-observer reliability and inter-eye variability}

For both conjunctival and corneal analyses, the observers had low bias, low mean percentage difference (variability), and high intra-class correlation, indicating strong agreement of measurements between observers. These results also serve as indicators of the reliability of IVCM in palpebral conjunctival and corneal immune cell quantification among trained personnel (Table 2). Interobserver variability ranged from $4.4 \pm 7.6 \%$ (SIC) to $9.5 \pm 2.7 \%$ (EIC) for conjunctival analysis and $11.9 \pm 5.1 \%$ (DC) for corneal analysis. ICC coefficients were strong for both conjunctival and corneal quantifications (ICC $>0.99, P<0.0001$ ).

There were no significant differences between the eyes of patients for any of the measured conjunctival (EIC $\mathrm{OD}=718.5 \pm 84.9$ cells $/ \mathrm{mm}^{2}$ vs $\mathrm{EIC} \mathrm{OS}=466.8 \pm 153.9$ cells $/ \mathrm{mm}^{2}, P=0.09 ;$ SIC OD $=95.0 \pm 30.0$ cells $/ \mathrm{mm}^{2}$ vs SIC OS $=92.9 \pm 69.8$ cells $\left./ \mathrm{mm}^{2}, P=0.29\right)$ and corneal immune cell parameters (DC OD $=83.8 \pm 40.9$ cells $/ \mathrm{mm}^{2}$, 

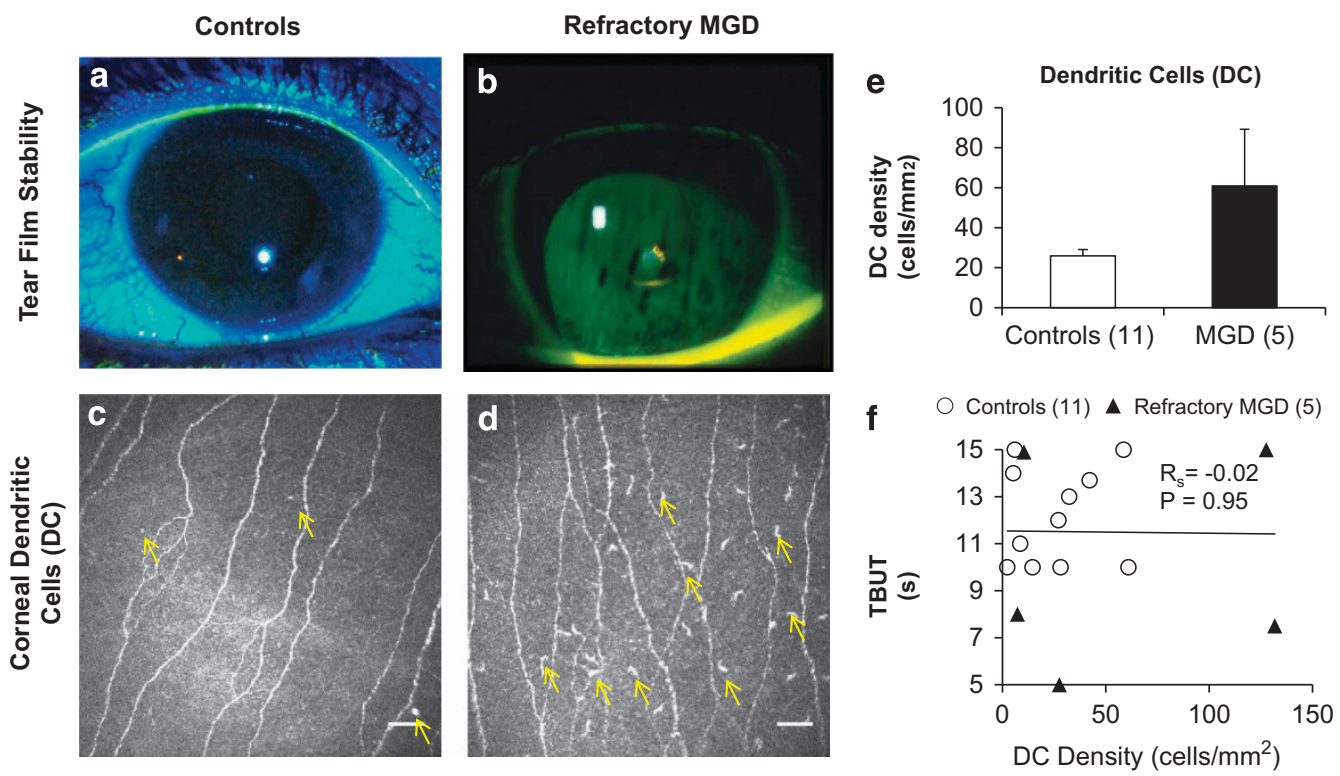

Figure 3 Corneal dendritic cells (DCs) in patients with persistent meibomian gland dysfunction (MGD)-associated dry eye symptoms. Representative slit-lamp photographs of tear film stability in healthy asymptomatic eyes (a) and patients with refractory MGD (b). As compared with healthy eyes (c, e; arrows indicate DCs), corneal in vivo confocal micrographs demonstrate no significant difference $(P=0.11)$ in the corneal DC densities of patients with refractory symptoms ( $\mathrm{d}$, e; arrows indicate DCs). Although there is a trend of increased corneal DC density in patients with persistent dry eye symptoms (c, d, e), the difference is neither statistically significant $(P=0.43)$ nor does it bear correlation with tear break-up time (TBUT; $\left.(\mathrm{f}), R_{\mathrm{S}}=-0.02, P=0.95\right)$. Results are reported as mean $\pm \mathrm{SEM}$. A probability value $(P)$ of less than 0.05 is considered statistically significant. The numbers in parentheses represent the number of patients per group. Scale bar represents $40 \mu \mathrm{m}$.

Table 2 Inter-observer reliability in palpebral conjunctival immune cell and corneal dendritic cell quantification using in vivo confocal microscopy (IVCM)

\begin{tabular}{|c|c|c|c|c|c|c|}
\hline Parameter & $\begin{array}{c}\text { Absolute difference } \\
\left.\text { (bias; cells } / \mathrm{mm}^{2}\right) \\
\text { (mean } \pm \text { SEM) }\end{array}$ & $\begin{array}{c}\text { Percentage difference }(\%) \\
(\text { mean } \pm \text { SEM) }\end{array}$ & $\mathrm{P}$ value ${ }^{\mathrm{a}}$ & $\begin{array}{c}\text { Intraclass correlation } \\
\text { coefficient }\end{array}$ & $\begin{array}{l}95 \% \text { Confidence interval } \\
\text { (upper, lower limits) }\end{array}$ & $\mathrm{P}$ value \\
\hline EIC & $10.9 \pm 6.4$ & $9.5 \pm 2.7$ & 0.67 & 0.997 & $0.992-0.999$ & $<0.0001$ \\
\hline SIC & $1.5 \pm 2.8$ & $4.4 \pm 7.6$ & 0.98 & 0.993 & 0.979-0.997 & $<0.0001$ \\
\hline DC & $3.9 \pm 1.7$ & $11.9 \pm 5.1$ & 0.75 & 0.991 & $0.967-0.997$ & $<0.0001$ \\
\hline
\end{tabular}

Palpebral conjunctival epithelial immune cell (EIC), palpebral conjunctival stromal immune cell (SIC), and central corneal dendritic cell (DC) densities were measured by two masked observers for both controls $(n=11)$ and patients $(n=5)$. Both observers had low bias and high correlation between their measurements for EIC, SIC, and DC. Results are reported as mean \pm standard error of mean (SEM) and a probability $(P)$ value of less than 0.0001 is

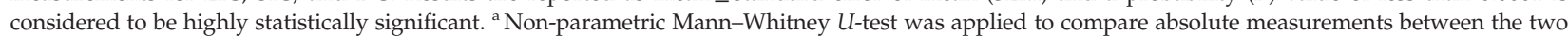
observers.

DC OS $=38.1 \pm 16.2$ cells $\left./ \mathrm{mm}^{2}, P=0.60\right)$, suggesting that data from both eyes may be taken together and averaged, as done in our methodology, without inducing statistical regression to the mean and skewed results.

\section{Qualitative features of the palpebral conjunctiva in patients with refractory MGD}

As compared with controls (Figure 4a,c, and e), numerous anatomic, immune cellular, and glandular changes were also qualitatively noted in MGD patients with persistent symptoms (Figure $4 \mathrm{~b} \mathrm{~d}$ and f). One patient had collections of immune cells within the palpebral conjunctiva, suggestive of conjunctiva-associated lymphoid tissue (Figure $4 \mathrm{~d}$ ). In addition, sub-epithelial fibrosis within the stroma (Figure $4 \mathrm{~b}$, filled arrowhead) and intraglandular immune cellular content (Figure $4 \mathrm{~b}$ and $\mathrm{f}$, empty arrowheads) were observed, the latter being associated with glandular dilatation (Figure $4 \mathrm{~b}$ and $\mathrm{f}$ ) unlike controls (Figure 4a and e).

\section{Discussion}

The disparity between symptoms and signs in DED, including MGD, has remained a clinical enigma until now. ${ }^{11-15}$ Herein, we report for the first time, to our 

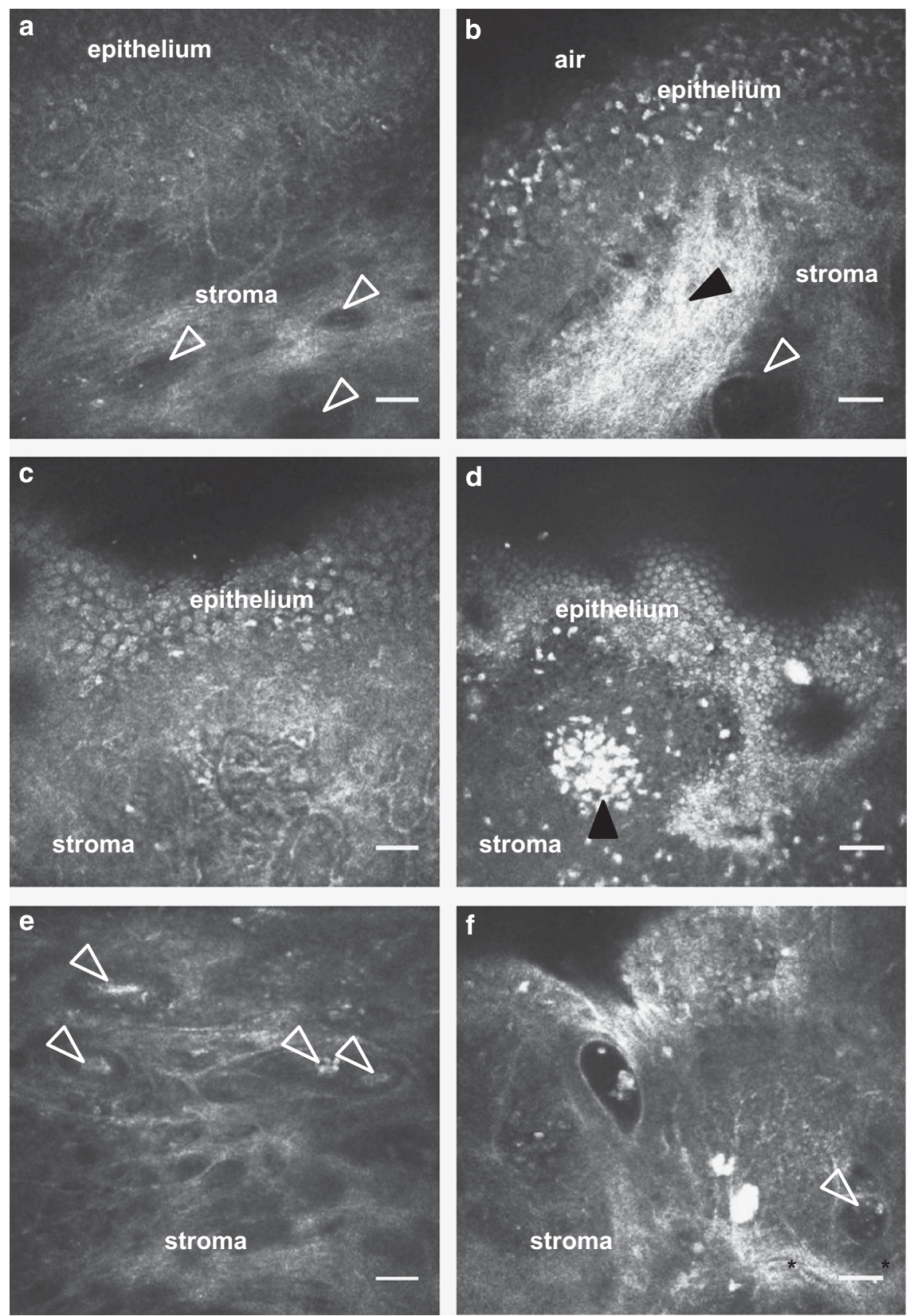

Figure 4 Qualitative features of the palpebral conjunctiva and associated glands in MGD-associated persistent dry eye symptoms using in vivo confocal microscopy (IVCM). Compared with the palpebral conjunctiva of healthy asymptomatic controls (a, c, e), IVCM micrographs of MGD patients with persistent symptoms (b, d, f) showed sub-epithelial fibrosis (b, filled arrowhead), dilated stromal glands with cellular content (b, f empty arrowhead), conjunctiva-associated lymphoid tissue (d, filled arrowhead). Some intrastromal glands with cellular content ( $f$, arrowhead) were surrounded by visible stromal blood vessels (f, asterisks). These intrastromal glandular structures with intraglandular content were seen at a depth of $\sim 200 \mu \mathrm{m}$ from the surface of palpebral conjunctival epithelium, suggestive of possible meibomian glands. Scale bar represents $40 \mu \mathrm{m}$.

knowledge, the presence of clinically non-apparent palpebral conjunctival inflammation in MGD patients with refractory symptoms following mechanical gland expression procedures. Our findings on IVCM, provide a possible explanation for the puzzling presence of symptoms in these patients who objectively improve upon clinical examination with treatment.
IVCM has allowed visualization of cellular detail in the evaluation of both the conjunctiva $a^{23,24,27,28}$ and cornea, ${ }^{29,30}$ which is comparable to and correlates strongly with ex vivo histological techniques such as immunohistochemistry ${ }^{31}$ and conjunctival impression cytology. ${ }^{32,33}$ This provision made by IVCM has allowed clinical research to spearhead, where previously obtaining 
human tissue samples of the conjunctiva was associated with worsening of ocular inflammation, ${ }^{34}$ and could not provide information about infiltration of inflammatory cells within and under the epithelium or the underlying architecture of the stroma and meibomian glands. ${ }^{32}$ Since the initial work on the use of IVCM in detection of immune cells in the normal palpebral conjunctiva and identification of presumed meibomian glands, ${ }^{27}$ more recent research in MGD and DED has allowed for the development of quantifiable parameters to assess and monitor both physiologic and disease states, ${ }^{23,25,35}$ including metrics to diagnose MGD such as acinar density. ${ }^{25}$ Our work presents an extension of the many applications of IVCM, specifically to detect clinically non-apparent palpebral conjunctival inflammation in MGD patients that suffer from refractory symptoms of discomfort, dry eye, and photophobia, despite objective improvement on clinical tests (TBUT, MGYLS) with an unremarkable slit-lamp examination. Such insight is likely to help the treating physician better understand and address the patient's symptoms, by considering additional treatment options such as anti-inflammatory therapy as necessary.

Inflammation of the ocular surface is typically detected at the slit-lamp biomicroscope employing a combination of methods including assessing integrity of the ocular surface epithelia using dyes (sodium fluorescein, lissamine green, and rose bengal), and detecting the presence of hyperemia and lid margin telangiectasia. The MGD patients with refractory symptoms discussed in our paper had been treated with mechanical measures and lubricants. Following treatment, they not only had an unremarkable slit-lamp examination, but also approached normal values for both TBUT and MGYLS scores, making the explanation and management of persistent dry eye symptoms challenging. On the contrary, the group of MGD patients who had improved symptoms and clinical signs had received systemic anti-inflammatory therapy (oral doxycycline), either alone or in combination with topical agents such as azithromycin or corticosteroids.

Currently, the role of topical corticosteroids in the treatment of MGD remains controversial, since inflammation may not always be clinically apparent, ${ }^{6}$ as exemplified by the cases presented in this paper. However, there is a growing body of evidence that suggests a beneficial role of anti-inflammatory therapy in MGD. ${ }^{6}$ Antibiotics that also have immunomodulatory functions such as macrolides (azithromycin) and tetracyclines (doxycycline) have yielded several reports of successful improvement in the signs and symptoms of MGD with topical and oral azithromycin, ${ }^{36-38}$ and oral doxycycline. ${ }^{36,39,40}$ All the refractory patients in our cohort had clinically non-apparent inflammation on IVCM, quantification of which demonstrated increased densities of both palpebral conjunctival epithelial and stromal immune cells as compared with MGD patients with improved symptoms. Corneal DC density was also elevated, which went undetected by routine slit-lamp examination. These findings are consistent with experimental models of DED, where both the conjunctiva and cornea were inflamed because of local production of inflammatory cytokines, such as interleukin- $1 \beta$ ), tumor necrosis factor- $\alpha$, and matrix metalloproteinase-9..$^{41}$ We speculate that the improved symptoms in MGD patients who had been treated with doxycycline and/or corticosteroids were in part due to the reduction in palpebral conjunctival epithelial immune cells, not made possible with mechanical and thermal gland expression procedures alone. Our speculation is supported by the highly significant correlation between EIC and OSDI found in this study.

Clinical examination in patients with MGD-associated refractory symptoms showed good improvement in both MGYLS and TBUT scores post treatment, normalizing to normal limits. However, their persistent and worsening symptoms remained unexplained. Given the findings by IVCM, and the improved symptoms in the antiinflammatory-treated MGD patients, it is tempting to speculate that patients with refractory dry eye symptoms may benefit from single or combination therapy of topical or systemic anti-inflammatory drugs discussed above. Based on our findings, we propose that it may thus lend a therapeutic advantage to assess both the baseline and follow-up immune status of the conjunctiva, toward ensuring optimal treatment of underlying clinically non-apparent inflammation that may cause continued discomfort despite a benign clinical examination. Our findings also indicate that mere patency of the meibomian gland orifices may not be sufficient for symptomatic relief, and that the extent of palpebral conjunctival tissue inflammation may contribute to patients' symptoms of ocular discomfort. This is especially interesting as we observed that OSDI scores were strongly and significantly correlated to both EIC and TBUT, which offers further insight into why simply improving TBUT is not sufficient for adequate symptomatic relief in evaporative dry eye patients. We therefore encourage that clinically nonapparent inflammation should be investigated and addressed in cases of persistent symptoms despite clinical improvement.

It is well established that meibomian glands, ${ }^{42}$ and the palpebral conjunctiva, ${ }^{43,44}$ are richly innervated with a supply of both autonomic and sensory nerves. These nerves not only release neurotransmitters and neuropeptides but also express cognate receptors, ${ }^{45,46}$ providing a channel for direct modulation of meibomian gland function and sensations of dryness, pain, and cooling. Some of these sensory neuropeptide receptors are also found on the neurovascular complex associated with meibomian gland acini, ${ }^{45,47}$ allowing circulating neuropeptides and cytokines to indirectly regulate both sensory stimuli and meibomian gland function. Given the 
propensity for autonomic-immune interactions following cytokine release by immune cells, ${ }^{48}$ it seems plausible that the presence of immune cells in the vicinity of palpebral conjunctival epithelium, substantia propria, and meibomian glands, as seen on IVCM, may stimulate these sensory nerves to trigger sensations of eye dryness and discomfort as reported by the patients presented in this paper. ${ }^{44,45,49}$ Thus, a completely accurate assessment of the health of the ocular surface, at least in some patients, may not be made based on slit-lamp examination alone given the microanatomy of the meibomian gland and associated architectural changes in disease, ${ }^{50,51}$ which could have therapeutic implications in these patients.

The International Workshop on Meibomian Gland Dysfunction recognized inflammation as a hallmark of MGD, and concluded that in order to advance the field, improved methods of clinical assessment of MGD were needed. ${ }^{6,52}$ Both the role of inflammation and methodology to assess inflammation in MGD have been evolving. With the application of IVCM, we were not only able to detect and quantify clinically non-apparent palpebral

conjunctival inflammation in refractory dry eye symptoms associated with MGD, but also identified abnormalities in the deeper layers of the palpebral conjunctival stroma and its glands (presumed meibomian glands) that indicated both active disease processes and potential damage from chronic inflammation. Our group is now studying these features in a larger cohort of MGD patients.

There are some limitations to our study: first, it is a pilot study with a smaller sample size. Nevertheless, this study warrants additional larger and longitudinal studies. Larger studies with increased statistical power may confirm the findings of our pilot study and further elucidate the role of SIC in patients with MGD. We believe that despite the lack of statistical significance, elevation in SIC may be an important indicator of the extent of immune cell infiltration and inflammation, which appeared to be greater in the group of patients with refractory symptoms, possibly due to a spillover from chronic epithelial inflammation. This hypothesis is supported by a positive and significant correlation between EIC and SIC in these patients $\left(R_{\mathrm{s}}=0.53, P=0.04, n=16\right)$. Second, not all patients in this study received the same treatment; two patients did not receive mechanical-thermal gland expression (LipiFlow, TearScience, Morrisville, NC, USA) in conjunction with manual digital gland expression. This does not seem to have induced bias since all patients remained symptomatic to varying degrees, despite objective clinical improvement regardless of the type of mechanical gland expression treatment received. The goal of this study was not to assess treatment responses, but rather to assess why these patients remained symptomatic despite objective improvement in signs.

In conclusion, our pilot study brought to attention several key findings: (a) MGD patients with refractory symptoms despite objective clinical improvement in TBUT and MGYLS and an unremarkable slit-lamp examination had clinically non-apparent inflammation with significantly elevated EIC and a near 20-fold increase in SIC on IVCM as compared with MGD patients with improved symptoms and signs, providing an explanation for symptom-sign disparity in this cohort; (b) symptom severity in MGD (OSDI score) was correlated to both EIC and TBUT, necessitating measures to address and normalize both parameters for adequate symptom relief; (c) mechanical procedures for MGD such as mechanical and mechanical-thermal gland expression do not address residual, clinically non-apparent inflammation in patients with persistent symptoms, who may benefit from anti-inflammatory therapy; (d) clinically non-apparent palpebral conjunctival inflammation can be detected and quantified using IVCM. Palpebral conjunctival IVCM is hence a valuable supplementary tool in the clinical assessment of persistent symptoms in MGD with the potential to guide physicians in customizing treatment plans according to the patient's tissue immune activation status.

\section{Summary}

\section{What was known before}

- Although most medical treatment regimens for MGD have a limited success rate, current advances with the advent of intraductal meibomian gland probing and thermal pulsation procedures have provided improved symptomatic relief.

- Despite advances in treatment, the issue of symptom-sign disparity, which is widespread in MGD-associated dry eye management, remains unaddressed.

- Symptom-sign disparity leads to sub-optimal symptomatic management of patients and increased patient dissatisfaction while there is objective clinical improvement.

\section{What this study adds}

- We employed in vivo confocal microscopy (IVCM) to investigate and address the unexplained symptom-sign disparity in a cohort of patients with refractory MGDassociated dry eye symptoms, despite clinical improvement following mechanical and/or thermal gland expression.

- We discovered that MGD patients with persistent symptoms, despite objective clinical improvement and a normal clinical slit-lamp examination, had active clinically non-apparent inflammation of the palpebral conjunctiva as compared with patients whose symptoms were successfully treated with anti-inflammatory regimens.

- These findings, for the first time, provide a probable explanation for refractory symptoms in MGD-associated dry eye disease, despite clinical improvement following mechanical procedures and an unremarkable slit-lamp examination, helping address the symptom-sign disparity in a subset of dry eye disease, suggesting a role for IVCMguided anti-inflammatory treatment in these patients.

\section{Conflict of interest}

Drs Hamrah and Qazi are inventors on a patent application regarding the utility of the diagnostic 
procedure and findings described in this report. The remaining authors declare no conflict of interest.

\section{Acknowledgements}

The work presented in this article was supported by $\mathrm{NIH}$ K08-EY020575 (PH), NIH L30-EY021919 (PH), Falk Medical Research Foundation (PH), and Research to Prevent Blindness Career Development Award (PH). The funding organizations had no role in the design or conduct of this research.

\section{References}

1 Viso E, Gude F, Rodriguez-Ares MT. The association of meibomian gland dysfunction and other common ocular diseases with dry eye: a population-based study in Spain Cornea 2011; 30(1): 1-6.

2 Bron AJ, Tiffany JM. The contribution of meibomian disease to dry eye. Ocul Surf 2004; 2(2): 149-165.

3 Rege A, Kulkarni V, Puthran N, Khandgave T. A clinical study of subtype-based prevalence of dry eye. J Clin Diagn Res 2013; 7(10): 2207-2210.

4 Nichols KK. The international workshop on meibomian gland dysfunction: introduction. Invest Ophthalmol Vis Sci 2011; 52(4): 1917-1921.

5 Schaumberg DA, Nichols JJ, Papas EB, Tong L, Uchino M, Nichols KK. The international workshop on meibomian gland dysfunction: report of the subcommittee on the epidemiology of, and associated risk factors for, MGD. Invest Ophthalmol Vis Sci 2011; 52(4): 1994-2005.

6 Geerling G, Tauber J, Baudouin C, Goto E, Matsumoto Y, $\mathrm{O}$ 'Brien $\mathrm{T}$ et al. The international workshop on meibomian gland dysfunction: report of the subcommittee on management and treatment of meibomian gland dysfunction. Invest Ophthalmol Vis Sci 2011; 52(4): 2050-2064.

7 Knop E, Knop N, Millar T, Obata H, Sullivan DA. The international workshop on meibomian gland dysfunction: report of the subcommittee on anatomy, physiology, and pathophysiology of the meibomian gland. Invest Ophthalmol Vis Sci 2011; 52(4): 1938-1978.

8 Maskin SL. Intraductal meibomian gland probing relieves symptoms of obstructive meibomian gland dysfunction. Cornea 2010; 29(10): 1145-1152.

9 Lane SS, DuBiner HB, Epstein RJ, Ernest PH, Greiner JV, Hardten DR et al. A new system, the LipiFlow, for the treatment of meibomian gland dysfunction. Cornea 2012; 31 (4): 396-404.

10 Greiner JV. Long-term (12-month) improvement in meibomian gland function and reduced dry eye symptoms with a single thermal pulsation treatment. Clin Experiment Ophthalmol 2013; 41(6): 524-530.

11 Cuevas M, Gonzalez-Garcia MJ, Castellanos E, Quispaya R, Parra Pde L, Fernandez I et al. Correlations among symptoms, signs, and clinical tests in evaporative-type dry eye disease caused by Meibomian gland dysfunction (MGD). Curr Eye Res 2012; 37(10): 855-863.

12 Johnson ME. The association between symptoms of discomfort and signs in dry eye. Ocul Surf 2009; 7(4): 199-211.
13 Nichols KK, Nichols JJ, Mitchell GL. The lack of association between signs and symptoms in patients with dry eye disease. Cornea 2004; 23(8): 762-770.

14 Sullivan BD, Crews LA, Messmer EM, Foulks GN, Nichols KK, Baenninger P et al. Correlations between commonly used objective signs and symptoms for the diagnosis of dry eye disease: clinical implications. Acta Ophthalmol 2012; 92(2): 161-166.

15 Baum JL. Evaporative dry eye-signs and symptoms don't mesh-and other thoughts. Ophthalmology 2010; 117(7): 1285-1286.

16 Ibrahim OM, Matsumoto Y, Dogru M, Adan ES, Wakamatsu $\mathrm{TH}$, Goto T et al. The efficacy, sensitivity, and specificity of in vivo laser confocal microscopy in the diagnosis of meibomian gland dysfunction. Ophthalmology 2010; 117(4): 665-672.

17 Dougherty BE, Nichols JJ, Nichols KK. Rasch analysis of the Ocular Surface Disease Index (OSDI). Invest Ophthalmol Vis Sci 2011; 52(12): 8630-8635.

18 Ozcura F, Aydin S, Helvaci MR. Ocular surface disease index for the diagnosis of dry eye syndrome. Ocul Immunol Inflamm 2007; 15(5): 389-393.

19 Schiffman RM, Christianson MD, Jacobsen G, Hirsch JD, Reis BL. Reliability and validity of the ocular surface disease index. Arch Ophthalmol 2000; 118(5): 615-621.

20 Ngo W, Situ P, Keir N, Korb D, Blackie C, Simpson T. Psychometric properties and validation of the Standard Patient Evaluation of Eye Dryness questionnaire. Cornea 2013; 32(9): 1204-1210.

21 Hamrah P, Sahin A, Dastjerdi MH, Shahatit BM, Bayhan HA, Dana R et al. Cellular changes of the corneal epithelium and stroma in herpes simplex keratitis: an in vivo confocal microscopy study. Ophthalmology 2012; 119(9): 1791-1797.

22 Matsumoto Y, Shigeno Y, Sato EA, Ibrahim OM, Saiki M, Negishi $\mathrm{K}$ et al. The evaluation of the treatment response in obstructive meibomian gland disease by in vivo laser confocal microscopy. Graefes Arch Clin Exp Ophthalmol 2009; 247(6): 821-829.

23 Wei A, Hong J, Sun X, Xu J. Evaluation of age-related changes in human palpebral conjunctiva and meibomian glands by in vivo confocal microscopy. Cornea 2011; 30(9): 1007-1012.

24 Villani E, Beretta S, Galimberti D, Viola F, Ratiglia R. In vivo confocal microscopy of conjunctival roundish bright objects: young, older, and Sjogren subjects. Invest Ophthalmol Vis Sci 2011; 52(7): 4829-4832.

25 Matsumoto Y, Sato EA, Ibrahim OM, Dogru M, Tsubota K. The application of in vivo laser confocal microscopy to the diagnosis and evaluation of meibomian gland dysfunction. Mol Vis 2008; 14: 1263-1271.

26 Cruzat A, Witkin D, Baniasadi N, Zheng L, Ciolino JB, Jurkunas UV et al. Inflammation and the nervous system: the connection in the cornea in patients with infectious keratitis. Invest Ophthalmol Vis Sci 2011; 52(8): 5136-5143.

27 Kobayashi A, Yoshita T, Sugiyama K. In vivo findings of the bulbar/palpebral conjunctiva and presumed meibomian glands by laser scanning confocal microscopy. Cornea 2005; 24(8): 985-988.

28 Villani E, Beretta S, De Capitani M, Galimberti D, Viola F, Ratiglia R. In vivo confocal microscopy of meibomian glands in Sjogren's syndrome. Invest Ophthalmol Vis Sci 2011; 52(2): 933-939. 
29 Mastropasqua L, Nubile M, Lanzini M, Carpineto P, Ciancaglini M, Pannellini T et al. Epithelial dendritic cell distribution in normal and inflamed human cornea: in vivo confocal microscopy study. Am J Ophthalmol 2006; 142(5): 736-744.

30 Erdelyi B, Kraak R, Zhivov A, Guthoff R, Nemeth J. In vivo confocal laser scanning microscopy of the cornea in dry eye. Graefes Arch Clin Exp Ophthalmol 2007; 245(1): 39-44.

31 Mayer WJ, Mackert MJ, Kranebitter N, Messmer EM, Gruterich M, Kampik A et al. Distribution of antigen presenting cells in the human cornea: correlation of in vivo confocal microscopy and immunohistochemistry in different pathologic entities. Curr Eye Res 2012; 37(11): 1012-1018.

32 Kojima T, Matsumoto Y, Dogru M, Tsubota K. The application of in vivo laser scanning confocal microscopy as a tool of conjunctival in vivo cytology in the diagnosis of dry eye ocular surface disease. Mol Vis 2010; 16: 2457-2464.

33 Kojima T, Matsumoto Y, Ibrahim OM, Sato EA, Dogru M, Tsubota K. In vivo evaluation of superior limbic keratoconjunctivitis using laser scanning confocal microscopy and conjunctival impression cytology. Invest Ophthalmol Vis Sci 2010; 51(8): 3986-3992.

34 Rolando M, Brezzo V, Calabria G. Ocular surface changes induced by repeated impression cytology. Adv Exp Med Biol 1994; 350: 249-254.

35 Efron N, Al-Dossari M, Pritchard N. In vivo confocal microscopy of the palpebral conjunctiva and tarsal plate. Optom Vis Sci 2009; 86(11): E1303-E1308.

36 Kashkouli MB, Fazel AJ, Kiavash V, Nojomi M, Ghiasian L. Oral azithromycin versus doxycycline in meibomian gland dysfunction: a randomised double masked open label clinical trial. Br J Ophthalmol 2014; 99(2): 199-204.

37 Foulks GN, Borchman D, Yappert M, Kim SH, McKay JW. Topical azithromycin therapy for meibomian gland dysfunction: clinical response and lipid alterations. Cornea 2010; 29(7): 781-788.

38 Igami TZ, Holzchuh R, Osaki TH, Santo RM, Kara-Jose N, Hida RY. Oral azithromycin for treatment of posterior blepharitis. Cornea 2011; 30(10): 1145-1149.

39 Yoo SE, Lee DC, Chang MH. The effect of low-dose doxycycline therapy in chronic meibomian gland dysfunction. Korean J Ophthalmol 2005; 19(4): 258-263.

40 Foulks GN, Borchman D, Yappert M, Kakar S. Topical azithromycin and oral doxycycline therapy of meibomian gland dysfunction: a comparative clinical and spectroscopic pilot study. Cornea 2013; 32(1): 44-53.

41 Luo L, Li DQ, Doshi A, Farley W, Corrales RM, Pflugfelder SC. Experimental dry eye stimulates production of inflammatory cytokines and MMP-9 and activates MAPK signaling pathways on the ocular surface. Invest Ophthalmol Vis Sci 2004; 45(12): 4293-4301.

42 Simons E, Smith PG. Sensory and autonomic innervation of the rat eyelid: neuronal origins and peptide phenotypes. J Chem Neuroanat 1994; 7(1-2): 35-47.

43 van der Werf F, Baljet B, Prins M, Ruskell GL, Otto JA. Innervation of the palpebral conjunctiva and the superior tarsal muscle in the cynomolgous monkey: a etrograde fluorescent tracing study. J Anat 1996; 189(Pt 2): 285-292.

44 Chung CW, Tigges M, Stone RA. Peptidergic innervation of the primate meibomian gland. Invest Ophthalmol Vis Sci 1996; 37(1): 238-245.

45 Seifert P, Spitznas M. Immunocytochemical and ultrastructural evaluation of the distribution of nervous tissue and neuropeptides in the meibomian gland. Graefes Arch Clin Exp Ophthalmol 1996; 234(10): 648-656.

46 Seifert P, Spitznas M. Vasoactive intestinal polypeptide (VIP) innervation of the human eyelid glands. Exp Eye Res 1999; 68 (6): 685-692.

47 Kirch W, Horneber M, Tamm ER. Characterization of Meibomian gland innervation in the cynomolgus monkey (Macaca fascicularis). Anat Embryol (Berl) 1996; 193(4): 365-375.

48 Kenney MJ, Ganta CK. Autonomic nervous system and immune system interactions. Compr Physiol 2014; 4(3): 1177-1200.

49 Hartschuh W, Weihe E, Reinecke M. Peptidergic (neurotensin, VIP, substance P) nerve fibres in the skin. Immunohistochemical evidence of an involvement of neuropeptides in nociception, pruritus and inflammation. Br J Dermatol 1983; 109(Suppl 25)): 14-17.

50 Obata H. Anatomy and histopathology of human meibomian gland. Cornea 2002; 21(7 Suppl): S70-S74.

51 Straatsma BR. Cystic degeneration of the meibomian glands. AMA Arch Ophthalmol 1959; 61(6): 918-927.

52 Tomlinson A, Bron AJ, Korb DR, Amano S, Paugh JR, Pearce EI et al. The international workshop on meibomian gland dysfunction: report of the diagnosis subcommittee. Invest Ophthalmol Vis Sci 2011; 52(4): 2006-2049. 